\title{
The Jaynes-Cummings model dynamics for nano-optical systems
}

\author{
S. Al-Awfi \\ Department of Physics, Taibah University, P. O. Box 30002, Madina, Saudi Arabia, \\ alawfi99@hotmail.com
}

Received: 17.03 .2012

\begin{abstract}
We investigate a two-level atom Jaynes-Cummings model and its dynamics for a nano-optical system. The optical system is assumed to be a hollow cylindrical waveguide with a circular cross section. Since the system is assumed to have nanoscale dimensions, the interaction process is strictly affected only by a single waveguide mode. The atom-mode coupling and the probability amplitudes are evaluated for different waveguide parameters as functions of the mode frequency.
\end{abstract}

Key words: nano-optical system, cylindrical waveguide, Jaynes-Cummings model

PACS: $03.75 . \mathrm{Be}, 37.30 .+\mathrm{i}$

UDC: 535.620 .3 .539 .182

\section{Introduction}

Recently, advances in both nano-fabrication technologies and production of high-intense tunable lasers have refreshed activities in atom optics [1-4]. They have led to significant progress in controlling neutral atoms using laser light $[5,6]$. In order to achieve accurate atomic interferometry using atomic waves, two major obstacles should be removed. The first is related to weak coherence of the atom waves. The concept of coherence of atomic matter-wave can be introduced as a direct analogy to that for the light waves, the light beam being replaced by the atomic beam [7]. However, one has to recognise many essential differences between the two kinds of coherence. Atoms have their internal structure and, moreover, the decay processes influence the coherence of the atomic beams. In addition, there are collisions between atoms and, in contrast to the optics, the free-space dispersion relations are different because atoms have mass, while the light is massless. Finally, atoms may be brought to rest while the light travels at a constant speed relative to all inertial observers [8]. The latter problem causes difficulty in selecting a particular mode to excite the atom. To remove these obstacles, we suggest using of a single-mode waveguide constructed in such a way as to allow only the fundamental mode to propagate, a method similar to the technique widely used in fibre optics [9].

In our previous work [10] we have been concerned with the theory of two-level atom and its interaction with a single quantised mode of a parallel-plate system described by a so-called Jaynes-Cummings model (JCM). It is well known that, due to relative simplicity of the system of parallel plates as a confining optical system, it is often used as a theoretical testing ground in quantum electrodynamics [11]. The present study is devoted to a more practical system, a cylindrical waveguide with a circular cross section [12]. We are interested in this system because (i) it is currently receiving attention in the context of the atom optics [1-8] and (ii) it is extremely important when manipulating atoms [9-12]. Finally, this kind of systems can confine atoms transversely in two dimensions and so have more capabilities for eliminating the problems of transverse diffusion which manifest themselves in the parallel-plate systems [11].

Ukr. J. Phys. Opt. 2012, V13, №3 125 
We will focus on the two-level atom JCM and its dynamics for a nanoscale twodimensionally confined optical system. The JCM has been suggested initially by Edwin Jaynes and Fred Cummings in 1963 [13] in order to study the relationships between the quantum theory of electromagnetic field and the semi-classical theory in describing decay rate of the emission.

The article is organised as follows. In section 2 , the optical system is primarily quantised, allowing the decay emission rate to be calculated. Variations of the emission decay rate for a single-mode operation of the system are explored in section 3. Section 4 deals with examining the JCM with and without dissipation in the optical system. Finally, section 5 contains the main conclusions and provides further comments on the subject.

\section{The dipole emission rate}

Our first objective is to outline a two-level atom interacting with the vacuum fields which are constrained by an infinitely hollow circular waveguide, as illustrated in Fig. 1. Such a system has a diameter $r=2 a$, where the longitudinal coordinate varies along the positive direction of the $z$ axis and its surfaces are postulated to be perfectly conducting. The total Hamiltonian for the atom plus field can be written as a sum of three terms,

$$
H=H_{A}+H_{F}+H_{\text {int }},
$$

where $H_{A}$ and $H_{F}$ are the zero-order Hamiltonians for the atom and the laser, respectively. They are given by

$$
\begin{gathered}
H_{A}=\frac{P^{2}}{2 M}+\hbar \omega_{0} \pi^{\dagger} \pi, \\
H_{F}=\hbar \omega_{0} a^{\dagger} a .
\end{gathered}
$$
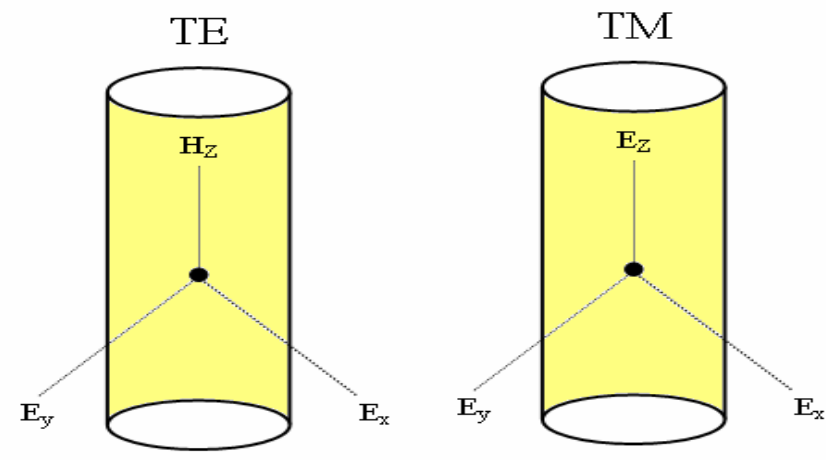

Fig. 1. Schematic drawing of hollow circular waveguide.

Here $\pi$ and $\pi^{\dagger}$ are the ladder operators for the two-level system, and $\mathbf{P}$ the centre-of-mass momentum operator (with $M$ being the mass and $\omega_{0}$ the dipole transition frequency). The operators $a$ and $a^{\dagger}$ entering $H_{F}$ are the annihilation and creation operators of the laser light, and $\omega$ is its frequency. The last term $H_{\text {int }}$ in Eq. (1) is the interaction Hamiltonian coupling the laser light to the two-level system in the electric dipole approximation. Explicitly we have

$$
H_{\text {int }}=-\boldsymbol{\mu} . \mathbf{E}(\mathbf{R}) \text {, }
$$


where $\mathbf{E}(\mathbf{R})$ is the electric field evaluated at the centre-of-mass position vector $\mathbf{R}$ and $\mu$ means the dipole moment vector operator

$$
\boldsymbol{\mu}=\boldsymbol{\mu}_{12}\left(\pi+\pi^{\dagger}\right)
$$

with $\mu_{12}$ being the transition dipole matrix element.

Quantisation of the electromagnetic fields inside such a waveguide can be given in terms of transverse electric ( $s$-polarised or $T E$ ) and transverse magnetic ( $p$-polarised or $T M$ ) modes satisfying the field boundary conditions at the inner surfaces. In this case the electric field can be written as

$$
\mathbf{E}(\mathbf{R}, t)=\sum_{\eta=s, p} \sum_{n} \sum_{m} \int d k_{\|}\left(a_{\eta}(k, n, m) F_{\eta}(k, n, m, \mathbf{R}, t)+c . c\right),
$$

where c.c stands for complex conjugate and $a_{\eta}(k, n, m)$ is the boson operator for the field mode of polarisation $\eta=s, p$, which is characterised by the integer quantum numbers $n, m$ and the parallel wave vector $k$ (with $n, m$ referring to the order of the excited mode). Finally, $F_{\eta}(k, n, m, \mathbf{R}, t)$ is the mode function satisfying field boundary conditions at the inner guide surfaces. Here standard electromagnetic boundary conditions apply such that the tangential components of the electric field vector and the magnetic field vector must vanish at every point on the inner cylinder surfaces, thus excluding all the electromagnetic fields from the interior. The travelling modes of such a system are well known $[12,14]$. They have been quantised for the case of multi-mode operation and involve summation of both the $s$-polarised (TE) and the $p$-polarised (TM) modes. In the case of single-mode operation, only the $T E_{11}$ mode can exist and its electric functions can be written as

$$
\begin{aligned}
\mathbf{E}^{s}(11, k, \mathbf{R}, t) & =i \omega_{11 k}^{s} a^{2} \mathrm{~N}_{0}^{S}(1.84)^{-1} \times \\
& {\left[1.84 a^{-1} J_{1}^{\prime}(1.84 r / a) \hat{\mathbf{e}}_{\phi}+i r^{-1} J_{1}(1.84 r / a) \hat{\mathbf{e}}_{r}\right] \exp ^{ \pm i \varphi} \exp ^{i\left(\omega_{k 11}^{s} t-k z\right)}, }
\end{aligned}
$$

where $k$ is the longitudinal wave vector, $J_{1}$ and $J_{1}^{\prime}$ respectively the first-order Bessel function and its first derivative, and $\omega_{11 k}^{5}$ the mode frequency given by

$$
\omega_{11 k}^{s}=c\left[k^{2}+\left(\chi_{11}^{s} / a\right)\right],
$$

where $\chi_{11}^{5}$ is the zero of the $J_{1}^{\prime}$ function and $\chi_{11}^{5}$ has the smallest root of all the Bessel function derivatives where the first root of $J_{1}^{\prime}$ is $\chi_{11}^{5}=1.84$. Finally, $\mathrm{N}_{0}^{S}$ in Eq. (1) is the $T E_{11}$ mode normalisation factor obtained as

$$
\mathrm{N}_{0}^{S}=\left[\frac{(1.84)^{2} \hbar}{\omega \varepsilon_{0} V a^{2}\left[(1.84)^{2}-1\right] J_{1}^{2}(1.84)}\right]^{1 / 2},
$$

where $V$ is the quantisation volume. The cut-off frequency and the cut-off wavelength of this lowest mode are

$$
\omega_{c}=1.84 c / a, \quad \lambda_{c}=2 \pi a / 1.84=3.4 a .
$$

With $a=0.5 \lambda$, the cut-off frequency and the cut-off wavelength of this particular mode are estimated as $\omega_{c} \approx 0.05 \mathrm{PHz}$ and $\lambda_{c} \approx 1.0 \mu m$, respectively. 
On the other hand, it is well known that when a quantum-mechanical system radiates spontaneously in a confined space, both the dipole emission rate and the energy levels experience changes. The dipole emission rate of a two-level atom located in a confined space becomes position-dependent. Rippin and Knight [14] have evaluated the dipole emission rate inside a hollow multi-mode circular waveguide. For a single-mode waveguide operation their evaluation may be reduced to the following form:

$$
\frac{\Gamma(r)}{\Gamma_{0}}=\frac{3 \lambda}{2 \pi a}\left[\Theta^{\varphi}(r)+\Theta^{r}(r)\right]
$$

where $\Gamma_{0}$ is the free-space dipole emission rate and $\lambda$ the free-space radiation wavelength. The $\Theta$ functions in Eq. (11) are defined via the relations

$$
\begin{gathered}
\Theta^{r}=\frac{2 a^{2}\left[\left(2 \pi a / \lambda_{0}\right)^{2}-(1.84)^{2}\right]^{-1 / 2}}{\left[(1.84)^{2}-1\right] J_{1}^{2}(1.84)} \frac{J_{1}^{2}(1.84 r / a)}{r^{2}}, \\
\Theta^{\varphi}=\frac{2(1.84)^{2}\left[\left(2 \pi a / \lambda_{0}\right)^{2}-(1.84)^{2}\right]^{-1 / 2}}{\left[(1.84)^{2}-1\right] J_{1}^{2}(1.84)} J_{1}^{\prime 2}(1.84 r / a) .
\end{gathered}
$$

In Fig. 2, we plot changes in the dipole emission rate occurring across diameter of the waveguide. For this particular mode, we observe that the emission rate is equal to $3 \Gamma_{0}$ at the inner cylinder surfaces. This result disagrees with the well known value equal to $2 \Gamma_{0}$ when the dipole is assumed to be fixed along the normal direction and placed close to the flat single-plate surface [11]. This difference is due to curvature of the inner cylinder surface.

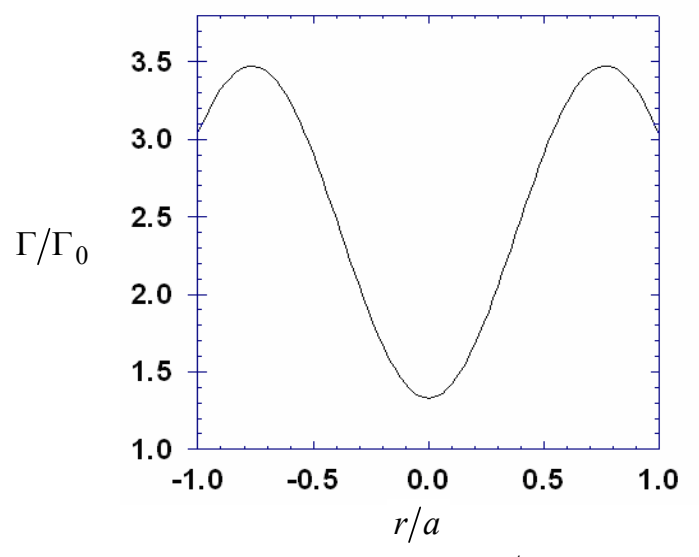

Fig. 2. Cross-section plot of $\Gamma / \Gamma_{0}$ parameter.

\section{Dynamics of the Jaynes-Cummings model}

As we have seen above, only the $T E_{11}$ mode exists for $a<\lambda / 2$ and the electric field of this mode is oriented normal to the inner cylinder surface. Then, given such a size of the optical system, it would exit the single-mode regime. This mode is actually quantised along the normal direction. The wave vector $k$ has a single continuous degree of freedom along the $z$ direction. Definitely, it is better to deal with than the case of the two-plate system which has two continuous degrees of freedom. To avoid this problem completely, subsequently we will take a quantum size of an empty 
closed cylindrical resonator [15]. Over and above, we will assume the inner surfaces to be perfectly conducting and so exclude all the electromagnetic fields from their interior.

In this situation, the standard electromagnetic boundary conditions apply such that the tangential components of the electric and magnetic field vectors must vanish at every point on the inner surfaces. Consequently, the energy injected during a very short time can remain stored inside the optical system for a very long time, when compared to the period, which means a very high quality factor Q. In addition to this interesting intrinsic property, the closed optical system, in general, is very significant for the theory of optical restriction of individual atoms by the mechanical forces associated with single photons. This kind of restriction corresponds to elementary quantum systems that are well isolated from the environment. Nonetheless, many experimental studies have recently been devoted to the open geometries as a suitable environment for the atom optics $[8,11,12]$. These geometries have clear advantages when it comes to loading the trap and, possibly, further manipulating the atoms (e.g., cooling or mode selecting), but they suffer from the problem that it is relativity easy for the atoms to escape. On the other hand, the closed geometries confine the atom in all directions and a particular mode can be excited from a tiny hole at an appropriate location on the outer surfaces. This hole may be connected with a laser source directly or by a waveguide, the latter being a common method for coupling modes in the closed geometries.

As a result of interaction of the two-level atom with the single-mode quantised field of a single-mode circular waveguide, the atom acquires an upper state $|2\rangle$ and a ground state $|1\rangle$, with the frequencies $\omega_{2}$ and $\omega_{1}$ and the probability amplitudes $C_{2, \ell}$ and $C_{2, \ell+1}$. This system is coupled to the single mode with the frequency $\omega$ and the quantity $\ell$ of photons. The Rabi frequency $\Omega_{s}$ for this case is replaced by $-2 g \sqrt{\ell+1}$. In the rotating-wave approximation, the system is described by the Jaynes-Cummings Hamiltonian [13], while the equations of motion for the probability amplitudes become

$$
\begin{aligned}
& i \dot{C}_{2, \ell}(t)=\left(\omega_{2}-\ell \omega\right) C_{2, \ell}(t)+g_{21} \sqrt{\ell+1} C_{1, \ell+1}(t), \\
& i \dot{C}_{1, \ell+1}(t)=\left(\omega_{1}-(\ell+1) \omega\right) C_{1, \ell+1}(t)+g_{12} \sqrt{\ell+1} C_{2, \ell}(t),
\end{aligned}
$$

where $g_{i j}=g_{j i}^{*}=g=\mathbf{d}_{i j} \mathbf{E} / \hbar$ is the mode-atom coupling between the states $i$ and $j$, and for generality the atomic nucleus is taken at the position $r$, instead of the origin. We have a system of coupled first-order differential equations. Its exact solution could be obtained by different techniques. In this section we will present the solution of this system using the approach similar to that employed in our previous work [10]. Namely, we take a trial solution of the form

$$
C_{2, \ell}=\mathrm{X}(\ell, t) C_{1, \ell+1}
$$

where $\mathrm{X}$ is any continuous function of $\ell$ and $t$. Substituting Eq. (16) into Eqs. (14) and (15) and comparing the resulting equations for the probability amplitudes, we obtain for $X$ the Riccati differential equation,

$$
\dot{X}(\ell, t)+i \Delta_{12} X(\ell, t)-g_{12} \sqrt{\ell+1} X^{2}(\ell, t)=-i g_{21} \sqrt{\ell+1},
$$


where the field detuning is defined as $\Delta_{12}=\left(\omega_{2}-\omega_{1}\right)-\omega$. After solving this equation, one can demonstrate straightforward that the probability amplitudes are given by the relations [16]

$$
\begin{aligned}
& C_{2, \ell}(t)=\frac{1}{g_{12}} \sqrt{\ell+1}\left[A\left(\Delta_{21}+\mu\right) \exp \left(\frac{i \mu}{2}\right)+B\left(\Delta_{21}-\mu\right) \exp \left(-\frac{i \mu}{2}\right)\right] \exp \left(-\frac{i \theta t}{2}\right), \\
& C_{1, \ell+1}(t)=[A \exp (i \mu t / 2)+B \exp (-i \mu t / 2)] \exp (-i \theta t / 2),
\end{aligned}
$$

with $\theta=(2 \ell+1) \omega+\omega_{2}+\omega_{1}$ and $\mu=\sqrt{\Delta_{12}^{2}+4(\ell+1)\left|g_{12}\right|^{2}}$.

The integration constants $A$ and $B$ are determined from the initial conditions of the problem. The above approach gives the well known exact solution of the two-state atom problem. Eqs. (18) and (19) can be used to evaluate the probabilities and the population inversion. For the coherent states, the amplitude $|\alpha\rangle$ of the probability that the photons are in the field at $t=0$ is given by (see $[17,18])$

$$
\begin{aligned}
& p_{\ell}=\left|C_{2, \ell}(0)\right|^{2}=\frac{\langle\ell\rangle^{\ell}}{\ell !} \exp (-\langle\ell\rangle), \\
& \langle\ell\rangle=|\alpha|^{2} .
\end{aligned}
$$

The probability amplitude for the atom in the state $j(=2,1)$ may be obtained by tracing over the field degrees of freedom. It is found to be

$$
p_{j}=\sum_{\ell}\left|C_{2, \ell}(t)\right|^{2} .
$$

The probability amplitudes given in the above expressions have been evaluated numerically and the probability inversion $W_{21}=P_{1}-P_{2}$ is displayed in Fig. 3 as a dependence on the $g t$ parameter for different detuning values $\Delta_{21}$. The field is described by the coherent state with the Poisson distribution at the centre $\langle\ell\rangle=20$. The collapse and the revival phenomena persist again.

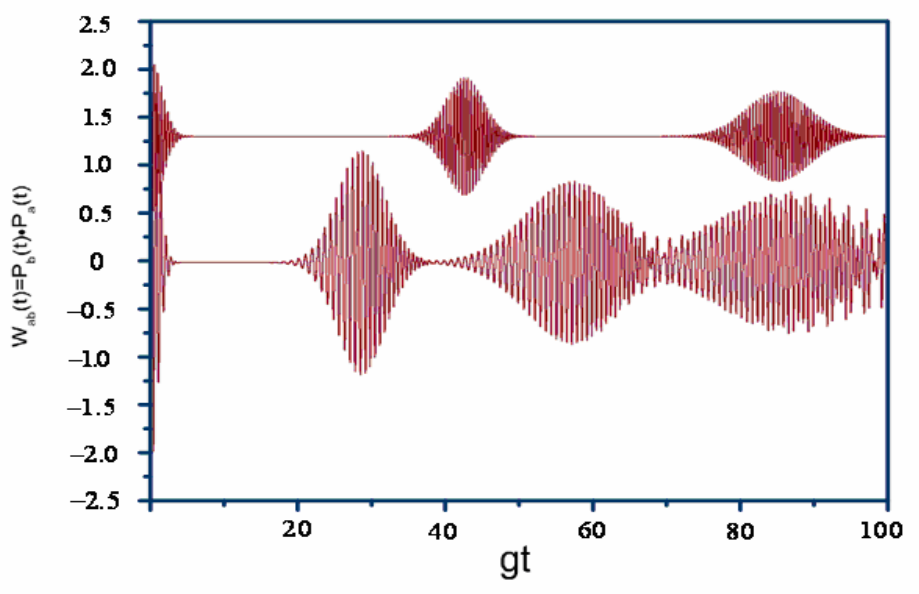

Fig. 3. Collapse and revival in the population inversion $W_{a b}(t)$ observed with evolving time at $\Delta_{21} / g=0.1,10$ and $\langle\ell\rangle=20$. 


\section{Influences of normal modes on the atom}

We suppose that a single atom is located at the point given by $z=0$ and $r=a / 2$ inside a nanoscale circular waveguide. In the absence of any external effects, the atom interacts with the vacuum fields which are at the present constrained by the nano-optical system. When $a<0.5<\lambda$, the possible decay modes are determined by Eqs. (11). It is clear that the atom interacts efficiently only with the unique mode of the frequency $\omega_{11 k}^{s}$. This atom-waveguide structure is characterised by two dissipative processes [19]: the first is associated with the coupling of the atom to the freespace background and the second one with the coupling of the waveguide mode to the environment, which can be neglected. With the same assumption, the corresponding equations of motion for the probability amplitudes $C_{2}(t)$ and $C_{1}(t)$ are

$$
\begin{aligned}
& \dot{C}_{2, \ell}(t)=\left[-i\left(\omega_{2}-\ell \omega\right)-\frac{\Gamma}{2}\right] C_{2, \ell}(t)-g_{21} \sqrt{\ell+1} C_{1, \ell+1}(t), \\
& \dot{C}_{1, \ell+1}(t)=-i\left(\omega_{1}-(\ell+1) \omega\right) C_{1, \ell+1}(t)-g_{12} \sqrt{\ell+1} C_{2, \ell}(t),
\end{aligned}
$$

where $\Gamma$ is the emission decay rate. Using the same technique, one can obtain the probability amplitudes for the initial conditions $C_{2}(0)=0$ and $C_{1}(0)=0$ as

$$
\begin{aligned}
C_{2}(t)= & \frac{-i g \sqrt{\ell+1}}{\sqrt{-\alpha+i \Gamma \Delta}} \times \\
& \times\left[\exp \left(\frac{1}{2} \sqrt{-\alpha+i \Gamma \Delta}\right)-\exp \left(-\frac{1}{2} \sqrt{-\alpha+i \Gamma \Delta}\right)\right] \exp \left(\frac{t}{2}(-\Gamma-i \theta)\right), \\
C_{1}(t)= & \frac{1}{2}\left[(1+A) \exp \left(\frac{1}{2} \sqrt{-\alpha+i \Gamma \Delta}\right)+(1-A) \exp \left(-\frac{1}{2} \sqrt{-\alpha+i \Gamma \Delta}\right)\right] \times, \\
& \times \exp \left(\frac{t}{2}(-\Gamma-i \theta)\right)
\end{aligned}
$$

where

$$
\begin{gathered}
A=\frac{\Gamma+i \Delta}{\sqrt{-\alpha+i \Gamma \Delta}}, \\
\alpha=-\Gamma^{2}+\mu^{2}, \\
\mu=\sqrt{\Delta^{2}+4(\ell+1)|g|^{2}} .
\end{gathered}
$$

The probability corresponding to the atom in the state $j(=2,1)$ is obtained by tracing over the field degrees of freedom. It becomes

$$
p_{j}=\sum_{\ell} p_{\ell}\left|C_{j}(t)\right|^{2}
$$

The solutions thus obtained are valid for the initial conditions $C_{2}(0)=0$ and $C_{1}(0)=0$, tough the problem can be solved for any other initial conditions. The behaviour of these quantities depends, however, on the quantum state of the radiation field through the term given by Eq. (20), which comprises the statistical properties of the field. In Fig. 4 we plot the probability $P_{1}(t)$ of the excited atomic state versus $g t$ for different decay rates $\Gamma$. Here exponentially decaying curve is 
peculiar for the weak-coupling regime $(\Gamma=100 g$, i.e. $\Gamma>>g)$ and damped oscillations correspond to the strong-coupling regime $(\Gamma=0.1 g$, i.e. $\Gamma<<g$, though in the presence of both the collapse and the revival phenomena. In the intermediate regime $(\Gamma=g)$, the damped oscillations are present only in the transient regime and the probability decreases exponentially with increasing time.

\section{Conclusions}

We have investigated dynamics of the JCM for the optical system in the shape of a hollow cylindrical waveguide with a circular cross section. This optical system can be fabricated for both the multi-mode and single mode cases of operations. The multi-mode regime is relatively easy for fabricating and it can be treated using the ray optics [20]. At the same time, the single-mode one is harder to fabricate, the coupling of light (and atoms) is stronger, and the ray-optics approximation breaks down. Then one must use the wave-optics approach. In the wave optics, one should assume that the wave is confined inside the hole of the waveguide, with a standing-wave pattern occurring in the lateral direction, the wave amplitude falling to zero at the hole edge, and an integral number of half-wavelengths fitted into the hole width [20].

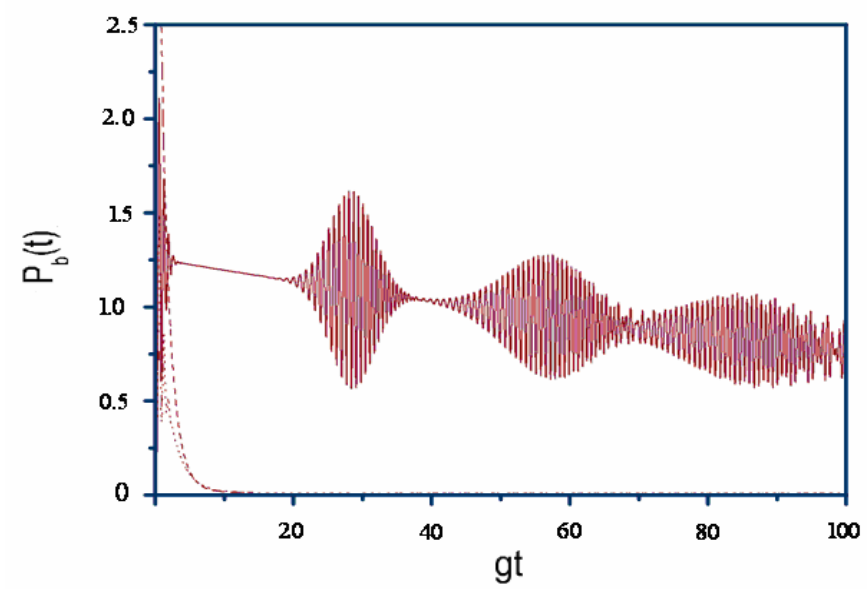

Fig. 4. Probability $P_{b}(t)$ as a function of time for the case of $\Delta_{21} / g=0.1,\langle\ell\rangle=20$ and different $\Gamma$ values: $\Gamma=0.01 g$ (solid line); $\Gamma=g$ (dotted line); $\Gamma=100 g$ (dashed line).

It is worthwhile that most of the recent works have concentrated on multi-mode operation, when the dipole emission rate approaches its free-space value. Some work has also been done for the case of sub-wavelength dimensions of the waveguide, where the dipole emission rate can only be mediated by a few feasible modes [10-12]. The present study considers a pure single-mode operation which allows propagation of only one mode, when the diameter of hole of the waveguide is taken to be of the order of $2-10 \mu \mathrm{m}$.

We have obtained the variations of the dipole emission rate under the condition that the atom is located at the point $(r=2 / a, z=0)$ inside the optical system. Next, the probability amplitudes have been evaluated numerically, the probability inversion $W_{21}=P_{1}-P_{2}$ has been studied depending on the $g t$ parameter for different values of detuning, and the field has been described by a coherent state with the Poisson distribution at the centre $\langle\ell\rangle=20$. We note that the collapse 
and the revival phenomena persist again. Finally, we have discussed basic aspects of the emission decay in this cavity for different coupling regimes.

Notice that we have focused only on the two-dimensional waveguides characterised by two distinct features: (i) the optical system is assumed to have a circular waveguide and (ii) the inner surfaces of the waveguide are taken to be perfect conductors. The related problem that can also be addressed is that of cylindrical atom waveguides, with the guide surfaces made of dielectrics characterised by dispersive dielectric functions which could exhibit losses. The theory considering such features should additionally accommodate the first type of the atom waveguide, namely, the evanescent mode guides which can now have a novel feature, nanoscale dimensions. Although the emission decay for the atoms in the dielectric waveguides has already been investigated [21], the details of the JCM for the two-level atoms and its dynamics, along the lines discussed in this study, have not yet been reported. The work along these lines is now in progress.

\section{References}

1. Andrews D. Structured light and its applications: An introduction to phase-structured beams and nanoscale optical forces. Burlington: MA Academic (2008).

2. Austin M and Chou S, 2004. Response to "Comment on "Fabrication of a molecular selfassembled monolayer diode using nano-imprint lithography" Nano Lett. 4: 535.

3. Akiba E, Enoki H and Nakamura Y, 2004. Nano scale structure such as nano-size crystallites and defects can be found in conventional hydrogen absorbing alloys. Mater. Sci. Eng. B. 108: $60-66$.

4. Wilk T, Webster S, Kuhn A and Rempe G, 2007. Single-atom single-photon quantum interface. Science. 317: 488-493.

5. Al-Awfi S, 2011. Atomic decay rate enhancement inside a cylindrical quantum nanotube. Int. J. Nanomanuf. 4: 84-89.

6. Lembessis V, Al-Awfi S, Babiker M and Andrews D, 2011. Surface plasmon optical vortices and their influence on atoms. J. Opt. 13: 064002.

7. Marksteiner S, Savage C, Zoller P and Rolston S, 1994. Coherent atomic waveguides from hollow optical fibers: quantized atomic motion. Phys Rev. A. 50: 2680-2690.

8. Dowling J and Gea-Banacloche J, 1996. Evanescent light-wave atom mirrors, resonator, waveguides and traps. Adv. Atom. Mol. Opt. Phys. 37: 1-94.

9. Al-Awfi S, Bowaaneh M, and Elabbar A, 2005. Theoretical analysis of channeling atom through a mono-mode hollow optical fiber. Indian J. Phys. 79(4): 385-390.

10. Bougouffa S and Al-Awfi S, 2009. The dynamics of the Jaynes-Cummings model in nanostructures. Physica Scripta. T134: 014011.

11. Al-Awfi S and Babiker M 1998. Atom dynamic between two conducting plates. Phys Rev. A. 58: $2272-2281$.

12. Al-Awfi S and Babiker M, 2000. Atomic motion in hollow sub-micron circular cylinder. Phys. Rev. A. 61: 033401.

13. Jaynes E and Cummings F, 1963. Comparison of quantum and semiclassical theory with applications to beam maser. Proc. IEEE. 51: 89-99.

14. Rippin $M$ and Knight $P, 1996$. Modified spontaneous emission in cylindrical micro cavities wave guiding and distributed Bragg reflecting structures. J. Mod. Opt. 47: 807-832.

15. Al-Awfi S, Bougouffa $S$ and Bawa'aneh $M, 2009$. Dipole emission rate inside a nano quantum dot resonator. Int. J. Nanomanufacturing, 4: 92-96. 
16. Kamli A and Bougouffa S, 2002. The dynamics of Jaynes-Cummings model in the presence of photonic crystals. J. Mod. Opt. 49: 897-904.

17. Loudon R. The quantum theory of light. Oxford, New York: Oxford Univ. Press (2000).

18. Scully M and Zubairy S. Quantum optics Cambridge: Cambridge Univ. Press (1997).

19. Meystre $P$ and Sargent M. Elements of quantum optics. Berlin Heidelberg, New York: Springer-Verlag (1999).

20. Senior J. Optical fibre communication principle and practices. Hemel Hempstead: Prentice Hall (1992).

21. Banyard G, Bennett C and Babiker M, 2002 Enhancement of energy relaxation rates near metal-coated dielectric cylinders. Opt. Commun. 207: 195-200.

S. Al-Awfi, 2012. The Jaynes-Cummings model dynamics for nano-optical systems. Ukr.J.Phys.Opt. 13: 125 - 134.

Анотація. В роботі досліджено дворівневий атом Джейнса-Каммінгса та його динаміка 6 нано-оптичній системі. Припускалось, що оптична система складається з порожніх циліндричних хвилеводів з круглим поперечним перерізом. Оскільки система вважається нано-розмірною, то на процес взаємодії впливає тільки одна хвилеводна мода. Оцінено атомно-модовий зв'язок $і$ амплітуди ймовірності для різних параметрів хвилеводу, як функиї частоти моди. 